\title{
Preventive practices towards Sexually Transmitted Infection and its determinants among young people in Ethiopia: a protocol for a systematic review and Meta-analysis
}

\section{Etsay Woldu Anbesu ( $\nabla$ etsaywold@gmail.com )}

Department of Public Health, College of Medical and Health Science, Samara University https://orcid.org/0000-0002-4532-6720

\section{Setognal Birara Aychiluhm}

Samara University

Mussie Alemayehu

Mekelle University College of Health Sciences

\section{Protocol}

Keywords: pooled prevalence, determinants, preventive practice, young people, sexually transmitted infection, Ethiopia

Posted Date: September 24th, 2021

DOI: https://doi.org/10.21203/rs.3.rs-737441/v4

License: (c) (i) This work is licensed under a Creative Commons Attribution 4.0 International License. Read Full License 


\section{Abstract \\ Background}

Globally, about 178.5 million new cases of curable sexually transmitted infections occur among young people aged 15-24 years. There are fragmented and inconsistent findings on preventive practices of sexually transmitted infection and there is no national representative data on preventive practices of sexually transmitted infection in Ethiopia. Thus, this systematic review and meta-analysis aimed to estimate the pooled prevalence of good preventive practices of sexually transmitted infection and identify its determinants among young people in Ethiopia.

\section{Methods}

The Preferred Reporting Items for Systematic review and Meta-analyses (PRISMA) guideline was employed to develop the review protocol. Databases such as PubMed, CINAH, Google, and Google Scholar were used to search published and unpublished studies from May $15-12$ August/2021. Joanna Briggs Institute Meta-Analysis of Statistics Assessment and Review Instrument will be used to assess the quality of the studies. Statistical heterogeneity will be checked using $1^{2}$ statistics. Subgroup analyses and meta-regression will be done based on regions to identify the sources of heterogeneity at a p-value $<0.05$. The statistical analysis will be done using STATA version-14 software. A random-effects model will be employed to estimate the pooled prevalence and identify determinants of preventive practices of sexually transmitted infections.

\section{Discussion}

Young people have a high unmet need for sexual and reproductive health services and poor preventive practices toward sexually transmitted infection. Despite different interventions implemented to enhance the preventive practice of sexually transmitted infection among young people, the problem is still a challenge in low-income countries, including Ethiopia

\section{Introduction}

Sexually transmitted infections (STIs) are diseases like gonorrhea, syphilis, chancroids, lymphogranuloma venerum, and more than 30 different bacteria, viruses, and parasites. STIs can be curable and incurable. The curable STIs include gonorrhea, syphilis, trichomonas, and chlamydia. The incurables STIs include the herpes simplex virus, hepatitis B, HIV, and human papillomavirus (HPV). The STIs transmit through sexual contact such as vaginal, anal, and oral sex. It can also spread through nonsexual means via blood or blood products and mother to child during pregnancy, childbirth, and breastfeeding. The common symptoms of or syndrome of STI include urethral discharge, vaginal 
discharge, genital ulcer, lower abdominal pain, inguinal bubo, neonatal conjunctivitis, and scrotal swelling [1-4]

Globally, about 357 million new cases of curable sexually transmitted infections occur among people aged 15-49 years, and over half of them are between $15-24$ years $[5,6]$. Every day, more than 1 million sexually transmitted infections (STIs) are acquired [1]. STIs are health threats to adolescents and young people in developed and developing countries [4, 7-11]. In Ethiopia, though there lack of surveillance data, a self-reported prevalence of STIs among youth aged $15-24$ years range from $1 \%$ to $3 \%$ [12]. STIs can have serious consequences include risk of HIV infection, stillbirth, neonatal death, low birth weight, sepsis, pneumonia, and neonatal conjunctivitis or blindness [1].

The age of young people by itself is a risk for many factors. It is a critical developmental period where youth begin to know and explain their sexual values and experimenting with sexual behaviors. They are at high risk for sexually transmitted infection (STI), unplanned pregnancy, abortion, low school performance, psychosocial problems, and economic crisis $[1,13]$. Moreover, rapid reproductive maturity among young people could lead to early sexual initiation and unsafe sex with the reluctance to use contraceptives methods [14-17].

In addition, factors such as multiple sexual partners, engaging in risky sexual activities, sex without a condom, sex with a commercial sex worker and older partners, consumption of alcohol and illicit drugs, cultural, religious, peer pressure, watching pornography, being single, nondisclosure of HIV status, conflicts between couples and families affect young people risky sexual behavior (RSB) $[4,8,10,18-24]$.

In Ethiopia, despite STIs remains one of the sustainable development goal agendas [25] and the development of national guidelines for the management of sexually transmitted infections using a syndromic approach [26], there is a lack of attention and surveillance data on preventive practice STIs in young people [27]. Moreover, though studies had conducted in different parts of the country on the preventive practices of sexually transmitted infection (STIs) among young people, there is no national representative data on preventive practices of sexually transmitted infection. Thus, this systematic review and meta-analysis aimed to estimate the pooled prevalence of good preventive practices of sexually transmitted infection (STIs) and identify its determinants among young people in Ethiopia.

\section{Research question}

- What is the pooled prevalence of good preventive practices towards sexually transmitted infection among young people in Ethiopia?

- What are the determinants of preventive practices towards sexually transmitted infection among young people in Ethiopia? 


\section{Objectives}

- To determine the pooled prevalence of good preventive practices towards sexually Transmitted Infection among young people in Ethiopia

- To identify the determinants of preventive practices towards sexually transmitted infection among young people in Ethiopia

\section{Methods}

\section{Review protocol development}

The Preferred Reporting Items for Systematic review and Meta-analyses (PRISMA) guideline will be employed to develop the review protocol [28] and PRISMA-P 2015 checklist format will be used to report the review procedure [29] (Additional file 1).

\section{PECO search guide}

Population: Young people (10-24 years old) [30].

Exposure: exposures are determinants that increase or decrease the likelihood of preventive practice toward sexually transmitted infections.

Comparison: The reference group for each determinant in each study will be the comparison variable. It may include good knowledge versus poor, positive attitude versus negative, education versus no education, access to information versus no accesses, consistent use of condoms versus not, etc.

Outcome. The primary outcome variable is the overall prevalence of good preventive practices of sexually transmitted infections (STIs) among young peoples in Ethiopia. The secondary outcome variables is the determinants of preventive practices of sexually transmitted infections (STIS) among young people in Ethiopia. Preventive practices of sexually transmitted infection refer the use of at least in one of the following activities; condom use, faithfulness, absenteeism from sex, decrease sexual partners, avoid sex with prostitute etc.

\section{Data source and searching strategies}

Databases such as PubMed, CINAH, Google, and Google Scholar will be used to search published and unpublished studies from May 15-12 August/2021. The two authors (EW and SB) will retrieve the studies. In addition, a cross-reference search will be employed to add other related studies from the final included studies that may miss in the databases search. The search term or keywords will be used using Boolean operator and the search string will be adapted based on the specific requirement of the database to identify relevant studies. (Additional file 2). The retrieve studies will be exported to Endnote version 8 reference manager software [31]. 


\section{Eligibility criteria}

All observational studies (cross-sectional, case-control, and cohort) will be included in the systematic review and meta-analysis study. Studies that reported the prevalence of preventive practices of sexually transmitted infection (STIS) and its determinants among young people in Ethiopia will be included. Moreover, we will also include studies that reported only the overall prevalence of good preventive practices of sexually transmitted infections (STIs) or at least one of the component of preventive practices of sexually transmitted infections. Both institutional and community-based studies on the prevalence of preventive practices of STIs will be included. Studies that only address the qualitative approach will be excluded from the study. However, if studies examine both quantitative and qualitative study findings, only the quantitative results will be considered. Studies published other than the English language, expert opinions, conferences, national surveys, and case reports will be excluded from the study. Restrictions to the date of publication will not be made.

\section{Selection of studies}

The two authors (EW and SB) will independently screen the studies based on the titles and abstract. Duplicates, irrelevant titles, and abstracts studies will be removed from the citation manager. Further quality evaluation will be made for full-text studies, and studies that meet the criteria of quality measurement standards will be included in systematic review and meta-analysis. The discussion will be made with the third author (MA) to solve any disagreement among the two reviewers (EW and SB) during the review process. The selection process will be presented using a PRISMA flow diagram (Additional file 3).

\section{Quality assessment}

The Joanna Briggs Institute Meta-Analysis of Statistics Assessment and Review Instrument (JBIMAStARI) will be employed to assess the quality and validity of the study [32]. The quality assessment will focus on clear inclusion criteria, study subjects and setting, standard measurement criteria, exposure and outcomes measurement, and appropriate statistical analysis (Additional 4). The quality of the studies will be assessed independently by the two authors (EW and SB). Studies $50 \%$ and above of the quality scale score will be considered for the final systematic review and meta-analysis study. Differences among reviewers during the quality review will be further discussed by the third author (MA).

\section{Data extraction}

Data extraction template form on Microsoft Excel (2016) will be prepared. Before the beginning of the actual data extraction, the data extraction pilot will be done in the data extraction template form. The first author's name, publication year, the study area, study design, sample size, associated factors, odds ratio, and prevalence of the studies will be extracted from the studies. In addition, the logarithm and standard error (SE) of the prevalence and odds ratio will be calculated on Microsoft Excel template form. The two authors (EW and SB) will extract the data independently. The discussion will be mad for any difference 
with a third author (MA) to reach on consensus. Communication will be made with the corresponding author for studies, in case of missing data or incomplete reports.

\section{Data synthesis and statistical analysis}

A narrative synthesis of results will be made for the included studies. A summary table and graph will be presented to describe the characteristics of the included studies results. A random-effects model [33] will be employed to estimate the overall pooled prevalence of preventive practices of sexually transmitted infections (STIS) and identify its determinants among youths in Ethiopia. A 95\% $\mathrm{Cl}$ will be used to declare the statistical significance. Statistical heterogeneity will be checked using the Cochran Q test [34] and I ${ }^{2}$ statistics [35]. $\left.\right|^{2}$ values represent $25 \%$ low, $50 \%$ moderate, and $75 \%$ substantial heterogeneity. Subgroup analyses and meta-regression based on regions will be done to identify the sources of heterogeneity among the studies at $p$-value $<0.05$. Sensitivity analysis will be done to assess the effect of studies on the overall effect estimate. The presence of publication bias will be checked using funnel plot observation[36], Egger's, and Beggar's statistical tests [37].

\section{Discussion}

This systematic review and meta-analysis protocol aimed to estimate the pooled prevalence of preventive practices of sexually transmitted infection (STIs) and identify its determinants among young people in Ethiopia. Though young people are sexually active, they have an unmet need for sexual and reproductive health services. The common barriers in low and middle-income countries include lack of behavioral change and accessibility of services [38-40]. Despite different interventions implemented to enhance the preventive practice of sexually transmitted infection (STIs) among young people, the problem is still a challenge in low-income countries, including Ethiopia $[12,26]$.

As far as we know, there is no study finding on the pooled prevalence of good preventive practices of sexually transmitted infection (STIs) and its determinants among young people in Ethiopia. Thus, this systematic review and meta-analysis will help policymakers to develop appropriate interventions on preventive practices of sexually transmitted infection (STIs) in Ethiopia. This study protocol may have a limitation on heterogeneity between studies. We will only include observational studies design and articles published only in the English language.

\section{Abbreviations}

JBI-MASt-ARI: Joanna Briggs Institute Meta-Analysis of Statistics Assessment and Review Instrument; PRISMA: Preferred Reporting Items for Systematic review and Meta-analyses; STI: Sexually transmitted infections; HPV: Human papillomavirus; HIV: human immunodeficiency virus; RSB: Risky sexual behavior; PECO: population, exposure, compression and outcome.

\section{Declarations}


Acknowledgements

We would like to thank Samara University for the provision of free HINARY databases website, internet and library access.

\section{Author contribution}

EW designed and drafted the systematic review and meta-analysis protocol. All authors reviewed the protocol manuscript. Each author read and approved the final version of the protocol.

\section{Funding}

No founder

\section{Availability of data and materials}

The protocol manuscript was submitted with additional files as supplementary materials.

\section{Ethics approval and consent to participate}

N/A

\section{Consent for publication}

Not applicable.

\section{Competing interests}

The authors declare no competing interests.

\section{References}

1. WHO. Sexually transmitted infections (STIs). Fact Sheets; 2019.

2. Lazarus JV, et al. Systematic review of interventions to prevent the spread of sexually transmitted infections, including HIV, among young people in Europe. Croatian Med J. 2010;51(1):74-84.

3. Organization WH, Report on global sexually transmitted infection surveillance 2018. 2018.

4. Samkange-Zeeb FN, Spallek L, Zeeb H. Awareness and knowledge of sexually transmitted diseases (STDs) among school-going adolescents in Europe: a systematic review of published literature. BMC Public Health. 2011;11(1):1-12.

5. Organization $\mathrm{WH}$. Strengthening the health sector response to care, support, treatment and prevention for young people living with HIV: WHO/UNICEF consultation, 13-17 November 2006 Blantyre, Malawi. World Health Organization; 2008.

6. Organization WH. Global health sector strategy on sexually transmitted infections 2016-2021: toward ending STIs. World Health Organization; 2016. 
7. Chanakira $E$, et al. Factors perceived to influence risky sexual behaviours among university students in the United Kingdom: a qualitative telephone interview study. BMC Public Health. 2014;14(1):1-7.

8. Chen $\mathrm{M}$, et al., Comparison of sexual knowledge, attitude, and behavior between female Chinese college students from urban areas and rural areas: a hidden challenge for HIV/AIDS control in China. BioMed research international, 2016. 2016.

9. Goundry ALR, Finlay ER, Llewellyn CD. Talking about links between sexually transmitted infections and infertility with college and university students from SE England, UK: a qualitative study. Reproductive health. 2013;10(1):1-7.

10. Hong Z, et al. Contraceptive knowledge, attitudes and behavior about sexuality among college students in Beijing, China. Chin Med J. 2012;125(6):1153-7.

11. Matkins PP. Sexually transmitted infections in adolescents. North Carolina medical journal. 2013;74(1):48-52.

12. FMOH. FMOH Central Statistical Agency, Ethiopia demographic and health survey, Addis Ababa. 2016.

13. Netsanet F, Abebe M. Risky sexual behaviors and associated factors among male and female students in Jimma Zone preparatory schools, South West Ethiopia: comparative study. Ethiopian Journal of Health Sciences. 2014;24(1):59-68.

14. Derese A, Seme A, Misganaw C. Assessment of substance use and risky sexual behaviour among Haramaya University Students, Ethiopia. Science Journal of Public Health. 2014;2(2):102-10.

15. Fetene N, Mekonnen W. The prevalence of risky sexual behaviors among youth center reproductive health clinics users and non-users in Addis Ababa, Ethiopia: a comparative cross-sectional study. PloS one. 2018;13(6):e0198657.

16. Morris JL, Rushwan H. Adolescent sexual and reproductive health: The global challenges. International Journal of Gynecology Obstetrics. 2015;131:S40-2.

17. Temesgen G, Markos Y. Assessment of substance use and risky sexual behaviour among public college students in Bonga town, Southwest Ethiopia. Am J Biomed Life Sci. 2015;3(5):91-7.

18. Bakhoum AY, et al., Assessment of knowledge, attitude, and practice of risky sexual behavior leading to HIV and sexually transmitted infections among Egyptian substance abusers: a cross-sectional study. Advances in Public Health, 2014. 2014.

19. Gebremedhin AT, et al. Khat chewing and risky sexual behavior in Sub-Saharan Africa: a systematic review protocol. JBI Evidence Synthesis. 2013;11(12):59-67.

20. Girmay A, Mariye T. Risky sexual behavior practice and associated factors among secondary and preparatory school students of Aksum town, northern Ethiopia, 2018. BMC Res Notes. 2019;12(1):17.

21. Melchiorre MG, et al. Social support, socio-economic status, health and abuse among older people in seven European countries. PloS one. 2013;8(1):e54856. 
22. Mersha A, et al. Risky sexual behaviors and associated factors among preparatory school students in Arba Minch town, Southern Ethiopia. Journal of Public Health Epidemiology. 2018;10(12):429-42.

23. Negeri EL. Assessment of risky sexual behaviors and risk perception among youths in Western Ethiopia: the influences of family and peers: a comparative cross-sectional study. BMC Public Health. 2014;14(1):1-12.

24. Uchudi J, Magadi M, Mostazir M, A multilevel analysis of the determinants of high risk sexual behavior (multiple sexual partners) in sub-Saharan Africa. Social Research Methodology Centre Working Paper: Africa. London, UK: Department of Sociology, City University, 2010.

25. Nino FS. Sustainable Development Goals-United Nations. United Nations Sustainable Development; 2015.

26. FMOH. National guidelines for the management of sexually transmitted infections using syndromic approach 2015.

27. Alfvén T, et al. Global AIDS reporting-2001 to 2015: lessons for monitoring the sustainable development goals. AIDS Behav. 2017;21(1):5-14.

28. Moher $D$, et al. Preferred reporting items for systematic reviews and meta-analyses: the PRISMA statement. PLoS Med. 2009;6(7):e1000097.

29. Shamseer $\mathrm{L}$, et al., Preferred reporting items for systematic review and meta-analysis protocols (PRISMA-P) 2015: elaboration and explanation. Bmj, 2015. 349.

30. UNDESA, Definition of Youth. United Nations Department of Economic and Social Affairs. 2013.

31. Bramer W, Bain P. Updating search strategies for systematic reviews using EndNote. Journal of the Medical Library Association: JMLA. 2017;105(3):285.

32. Munn Z, Tufanaru C, Aromataris E. JBI's systematic reviews: data extraction and synthesis. AJN The American Journal of Nursing. 2014;114(7):49-54.

33. Berkey CS, et al. A random-effects regression model for meta-analysis. Statistics in medicine. 1995;14(4):395-411.

34. Cooper $\mathrm{H}$, Hedges LV, Valentine JC. The handbook of research synthesis and meta-analysis. Russell Sage Foundation; 2019.

35. Higgins JP, Thompson SG. Quantifying heterogeneity in a meta-analysis. Statistics in medicine. 2002;21(11):1539-58.

36. Liu JL. The role of the funnel plot in detecting publication and related biases in meta-analysis. EvidBased Dent. 2011;12(4):121-2.

37. Egger M, et al. Bias in meta-analysis detected by a simple, graphical test. Bmj. 1997;315(7109):62934.

38. Menna T, Ali A, Worku A. Effects of peer education intervention on HIV/AIDS related sexual behaviors of secondary school students in Addis Ababa, Ethiopia: a quasi-experimental study. Reproductive health. 2015;12(1):1-8. 
39. Newton-Levinson A, Leichliter JS, Chandra-Mouli V. Sexually transmitted infection services for adolescents and youth in low-and middle-income countries: perceived and experienced barriers to accessing care. Journal of Adolescent Health, 2016. 59(1): p. 7-16.

40. Seangpraw K, et al. THE EFFECT OF SEX EDUCATION AND LIFE SKILLS FOR PREVENTIVE SEXUAL RISK BEHAVIOURS AMONG UNIVERSITY OF STUDENTS THAILAND. Journal of Ayub Medical College Abbottabad. 2017;29(4):540-6.

\section{Supplementary Files}

This is a list of supplementary files associated with this preprint. Click to download.

- Additionalfile1.doc

- Additionalfile2.docx

- Additionalfile3.docx

- Additionalfile4.docx 